\title{
Study of Resistive Plate Chambers for the Alice dimuon spectrometer
}

\author{
R. Arnaldi, ${ }^{a}$ A. Baldit, ${ }^{b}$, N. Bastid ${ }^{b}$, G. Blanchard ${ }^{b}$, E. Chiavassa ${ }^{a}$, P. Cortese ${ }^{\text {a }}$, G. Dellacasa ${ }^{c}$, \\ N. De Marco a , P. Dupieux ${ }^{\text {b }}$, B. Espagnon ${ }^{\text {b }}$, J. Fargeix ${ }^{b}$, M. Gallio ${ }^{\text {a }}$, L. Luquin, ${ }^{d}$ F. Manso ${ }^{b}$, \\ V. Metivier ${ }^{\mathrm{d}}$, A. Musso ${ }^{\mathrm{a}}, \underline{\text { A. Piccotti }}{ }^{\mathrm{a}}$, A. Rahmani ${ }^{\mathrm{d}}, \mathrm{V}$. Ramillen ${ }^{\mathrm{b}}$, O. Roig $^{\mathrm{b}}$, L. Royer $^{\mathrm{b}}$, \\ E. Scomparin ${ }^{\text {a }}$, C. Soave ${ }^{a}$, E. Vercellin ${ }^{\text {a }}$
}

For the ALICE collaboration

a INFN Torino and Dipartimento di Fisica sperimentale dell'Universita' di Torino

'baboratoire de Phisyque Corpuscolaire de Clermont-Fd, IN2P3-CNRS-Universite' Blaise Plascal

'INFN Torino and Universita' del Piemonte Orientale "Amedeo Avogadro", Alessandria

${ }^{\mathrm{d}}$ Laboratoire de Physique Subatomic et des Technologies Associees, Nantes

The trigger system of the Alice dimuon spectrometer is based on RPC detectors. We present experimental results of a beam test about rate capability, time resolution and cluster size of single gap RPC detectors operating both in 'streamer mode' and in 'avalanche mode'. We have compared the performances of small chambers $(50 \mathrm{x} 50$ $\mathrm{cm}^{2}$ ) built with bakelite of different resistivity, from $\sim 3 \cdot 10^{9} \Omega \mathrm{cm}$ to $\sim 3 \cdot 10^{11} \Omega \mathrm{cm}$. For the low resistivity RPC we obtained a rate capability of several hundreds of $\mathrm{Hz} / \mathrm{cm}^{2}$ when it is operating in 'streamer mode', and of several thousands of $\mathrm{Hz} / \mathrm{cm}^{2}$ when it is operating in 'avalanche mode'.

\section{INTRODUCTION}

The ALICE experiment will study nucleusnucleus interactions at the LHC in order to investigate nuclear matter at extreme energy density where the formation of the Quark-Gluon-Plasma (QGP) is expected. The ALICE dimuon spectrometer is dedicated to the study of the production of $\mathrm{J} / \Psi$ and $\Upsilon$ resonances: the suppression of these particles is a signature of the transition of nuclear matter to the QGP [1]. The spectrometer measures the complete spectrum of heavy quark mesons, i.e. J/ $\Psi, \Psi^{\prime}, \Upsilon, \Upsilon^{\prime}$ and $\Upsilon^{\prime \prime}$, via their muonic decay in pp and heavy ion collisions [2]. The trigger detectors of the ALICE muon spectromer will be Resistive Plate Chambers (RPC) $[3,4]$ which are simple devices well suited to trigger purposes: fast response and good time resolution; futhermore they are industrially produced and suitable to cover large areas. For these reasons the RPC are in use in various experiments and they will be largely used at the LHC experiments for the muon trigger.
RPC tests on beam have been performed in order to optimize the detector's performances for the ALICE experimental conditions.

\section{ALICE REQUIREMENTS FOR THE RPCs}

The experimental layout of the ALICE dimuon spectrometer is shown in fig.1. The angular acceptance goes from $2^{\circ}$ to $9^{\circ}(2.5 \leq \eta \leq 4.0)$. The main elements of the spectrometer are: three absorbers (front absorber, beam shield and muon filter) which reduce the partile flux into the muon spectrometer, the dipole magnet with a field integral of $3 \mathrm{Tm}, 10$ planes of tracking chambers and 2 trigger stations, $\mathrm{MC} 1$ and $\mathrm{MC}$. The trigger stations are placed at the end of the spectrometer, respectively at $16 \mathrm{~m}$ and $17 \mathrm{~m}$ from the interaction point and cover an area of about $6 \times 6$ $\mathrm{m}^{2}$ with a $0.6 \times 0.6 \mathrm{~m}^{2}$ opening in the centre to accomodate beam pipe and shielding. Each trigger station is made of 2 planes of 18 single gap RPCs read-out on both sides by means of strips 


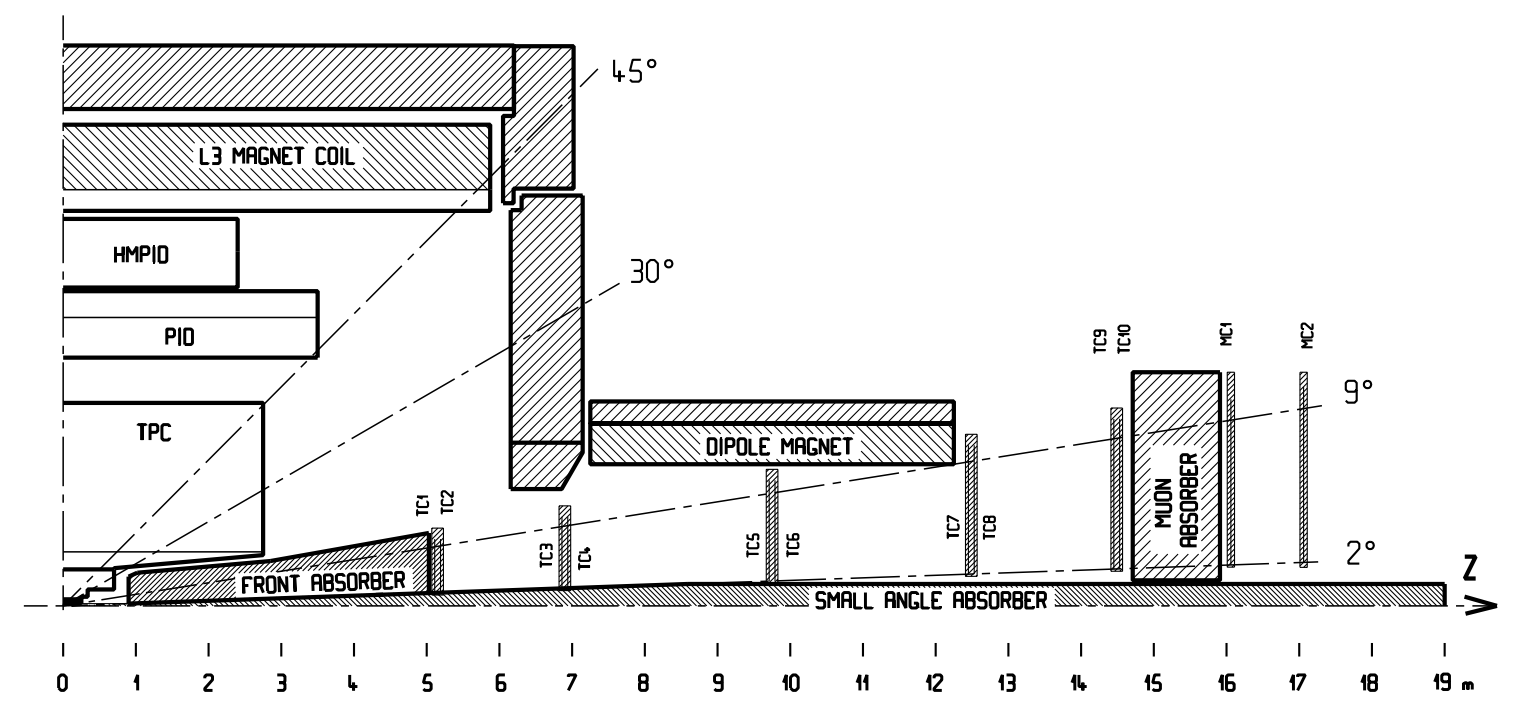

Figure 1. Schematic vew of the Alice dimuon arm

in the two orthogonal directions. In the bending plane the strip widths are 1-2-4 cm, going from the inner to the outer part of the trigger stations, instead in the non-bending plane, only two different strip widths are used: 2 and $4 \mathrm{~cm}$. With this configuration there are about 30000 read-out channels for the 4 RPC planes.

The trigger logic is based on transverse momentum cut in order to select high $\mathrm{p}_{t}$ muons coming from resonances decay $(\mathrm{J} / \Psi$ and $\Upsilon)$ from low $\mathrm{p}_{t}$ background (from $\pi$ and K decay). The first level trigger perfoms a loose $\mathrm{p}_{t}$ cut on the detected tracks, the second level trigger performs a more precise cut on the $\mathrm{p}_{t}$ and on the invariant mass of the dimuon, using the full information of the trigger chambers.

The trigger chambers of the dimuon spectrometer will operate in presence of an important background due to a large number of muons from pions, kaons, charm and beauty decay and to low energy particles coming out of the absorber and the beam shield. From FLUKA simulations [5], which take into account all these backgrounds, the maximum rate to be tolerated by the RPCs in ALICE will be $\sim 40 \mathrm{~Hz} / \mathrm{cm}^{2}$ in the Ca-Ca interaction at the LHC luminosity of $10^{29} \mathrm{~cm}^{-2} \mathrm{~s}^{-1}$ and $\sim 5 \mathrm{~Hz} / \mathrm{cm}^{2}$ in the $\mathrm{Pb}-\mathrm{Pb}$ interaction at the
LHC luminosity of $10^{27} \mathrm{~cm}^{-2} \mathrm{~s}^{-1}$.

The other requirements on the RPCs for the ALICE experiment are: efficiency $\epsilon \geq 95 \%$ for each plane, a time resolution of $\sim 2$ ns (r.m.s.) and a cluster size as close to 1 as possible in order to optimize the trigger selectivity.

\section{BEAM TEST}

In principle all the experimental requirements, presented in the previous section, could be satisfied by RPCs operating in streamer mode or in avalanche mode. The possibility to operate in streamer mode has been thoroughly investigated and compared to the avalanche operation mode during beam tests of RPC prototypes performed at the SPS at CERN.

As the electrode resistivity is a very important parameter for the rate capability of RPC operating in streamer mode, 3 small $\left(50 \times 50 \mathrm{~cm}^{2}\right)$ single gap RPCs with resistivity from $\rho=3.5 \cdot 10^{9} \Omega \mathrm{cm}$ to $\rho=3 \cdot 10^{11} \Omega \mathrm{cm}$ were studied. The low resistivity chamber $\left(\rho=3.5 \cdot 10^{9} \Omega \mathrm{cm}\right)$ was tested both in streamer mode and in avalanche mode: efficiency, cluster size and time resolution measurements were performed.

The RPCs operating in streamer mode were 


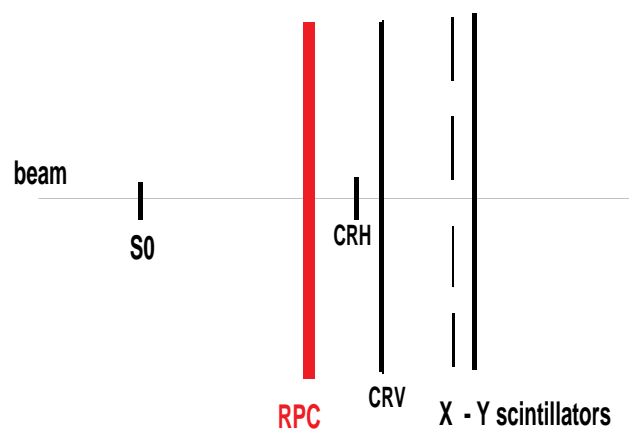

Figure 2. Schematic vew of the experimental setup for the beam test

fluxed with a gas mixture made up of: $49 \% \mathrm{Ar}$ $+40 \% \mathrm{C}_{2} \mathrm{H}_{2} \mathrm{~F}_{4}+7 \% \mathrm{C}_{4} \mathrm{H}_{10}+4 \% \mathrm{SF}_{6}$, according to the results obtained in previous laboratory test performed with cosmic rays [6]. The RPC operating in avalanche mode were fluxed with a gas mixture made up of: $95 \% \mathrm{C}_{2} \mathrm{H}_{2} \mathrm{~F}_{4}+3 \% \mathrm{C}_{4} \mathrm{H}_{10}+$ $2 \% \mathrm{SF}_{6}$, which gives a good efficiency $(\epsilon \geq 95 \%)$ for a large high voltage range $(\sim 500 \mathrm{~V})$ free from streamer contamination [7].

The RPC were tested separately on a defocussed pion beam of $120 \mathrm{GeV} / \mathrm{c}$ with a simple experimental set-up showed in fig.2. A large X-Y scintillator hodoscope of $40 \mathrm{x} 40 \mathrm{~cm}^{2}$ placed behind the RPC covers almost all the chamber surface. The 2 scintillators CRH and CRV define a small area $\left(2 \times 2 \mathrm{~cm}^{2}\right)$ in the center of the beam spot and are used to perform local efficiency measurements.

The strip signals were directly discriminated for the RPC operating in streamer mode, and they were amplified by a factor 300 before discrimination when the chamber is operating in avalanche mode. Constant fraction discriminators were used in order to perform a more precise time resolution measurement.

\subsection{Streamer mode results}

The RPC electrodes are made of bakelite planes whose resistivity is the main factor which affects the rate capability of the RPC , as mentioned before. In fact the time necessary to recharge the electrode surface after a spark is determined by

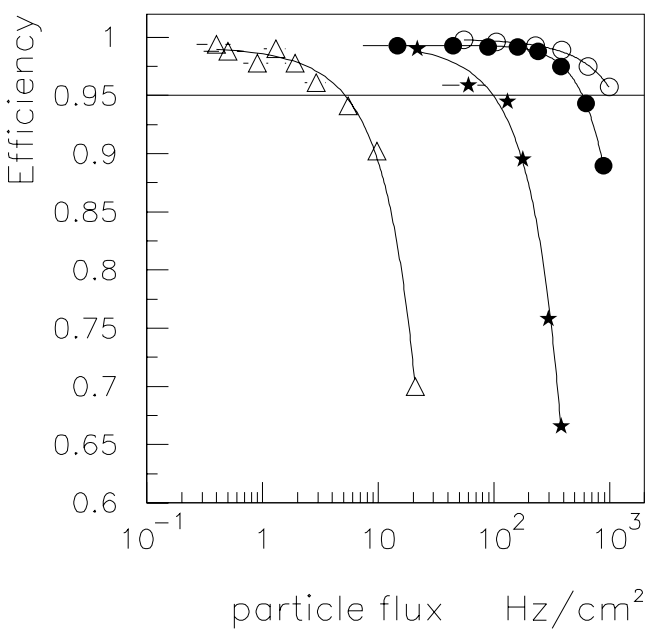

Figure 3. Efficiency as a function of particle flux for RPC of different resistivity operating in streamer mode:

- $\rho=3.5 \cdot 10^{9} \Omega \mathrm{cm} \quad \mathrm{HV}=9.4 \mathrm{KV}$

- $\rho=3.5 \cdot 10^{9} \Omega \mathrm{cm} \quad \mathrm{HV}=9 \mathrm{KV}$

* $\rho=6.0 \cdot 10^{10} \Omega \mathrm{cm} \quad \mathrm{HV}=9.2 \mathrm{KV}$

$\triangle \rho=3.0 \cdot 10^{11} \Omega \mathrm{cm} \quad \mathrm{HV}=9 \mathrm{KV}$.

the current that can flow across them and therefore is strictly related to the electrode resistivity. In fig. 3 is shown the RPC efficiency as a function of the particle flux for 3 different electrode resistivity. For each chamber the operation high voltage was choosen to be $\sim 200 \mathrm{~V}$ above the starting point of the efficiency plateau. The maximum flux tolerated by the RPC with a good efficiency $(\epsilon \geq 95 \%)$ goes from $\sim 5 \mathrm{~Hz} / \mathrm{cm}^{2}$ for the high resistivity chamber $\left(\rho=3.5 \cdot 10^{11} \Omega \mathrm{cm}\right)$ to $\sim 550 \mathrm{~Hz} / \mathrm{cm}^{2}$ for the low resistivity chamber $\left(\rho=3.5 \cdot 10^{9} \Omega \mathrm{cm}\right)$ : it increases in inverse proportion with the resistivity. The rate capability of the low resistivity chamber fulfill the Alice experimental requirements also with a large safety factor which has to be taken into account.

Fig. 4a shows an example of cluster size distribution for the low resistivity RPC equipped with $2 \mathrm{~cm}$ wide strips and operating at $9 \mathrm{KV}$ : a mean value of 1.1 strips was obtained. For the chamber equipped with $1 \mathrm{~cm}$ wide strips the measured cluster size mean value is 1.5 strips. 

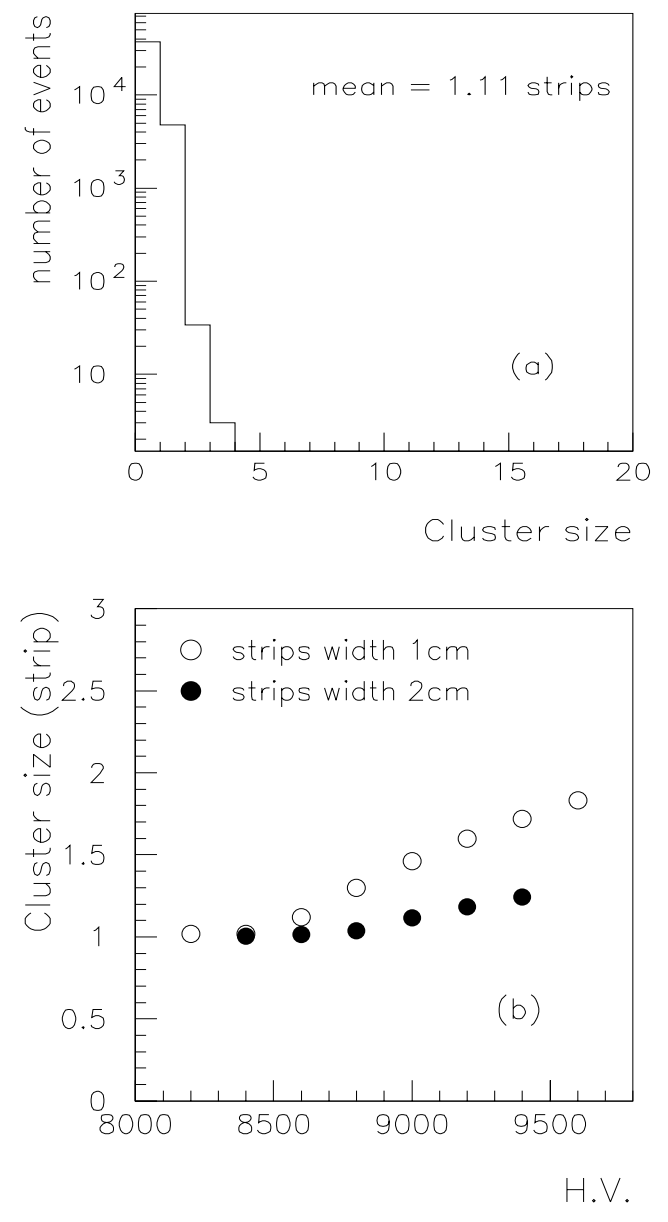

Figure 4. Cluster size for the low resistivity RPC operating in streamer mode:

(a) example of cluster size distribution for the chamber equipped with $2 \mathrm{~cm}$ wide strips.

(b) mean cluster size as a function of high voltage.

The mean cluster size increases slightly with the operating high voltage, as shown in fig. $4 \mathrm{~b}$, as the streamer charge increases with the operation voltage inside the gas gap.

On the other hand an increase in the operation high voltage improves the RPC time resolution as shown in fig.5b where the time distribution r.m.s. as a function of the operating voltage is presented. An example of the time distribution for low resistivity chamber at $9 \mathrm{KV}$ is shown in fig. 5a: it is evident that besides a narrow peak (r.m.s. 1ns)
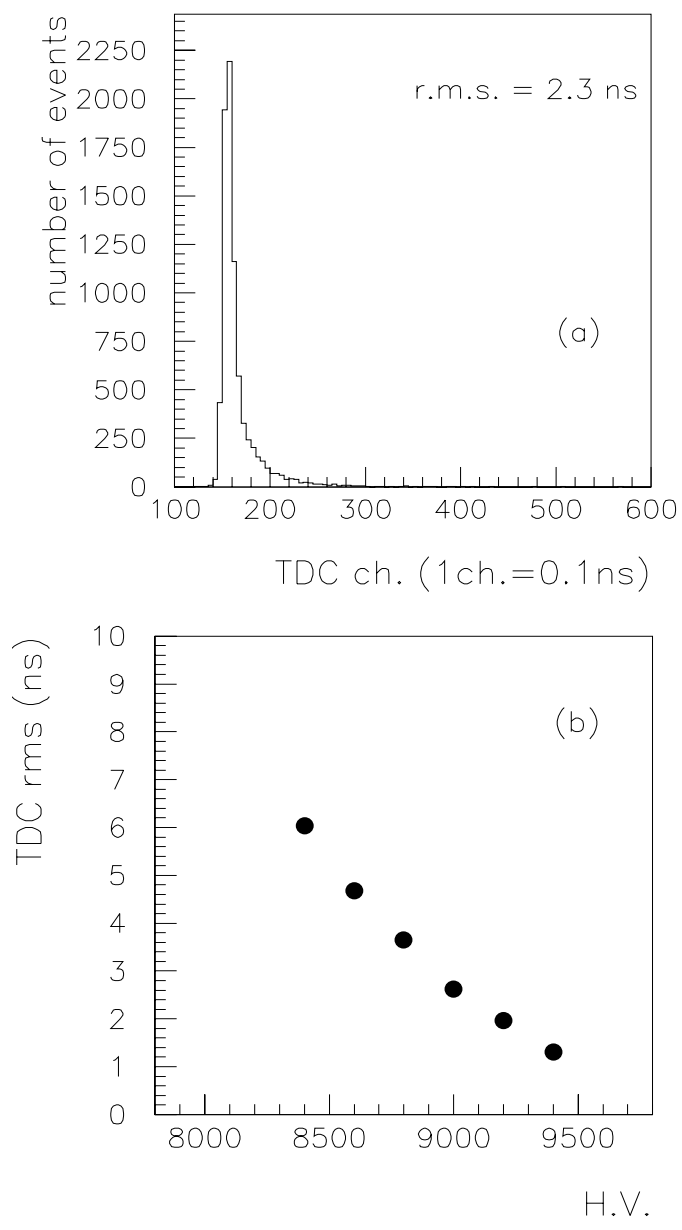

Figure 5. Time resolution for low resistivity RPC operating in streamer mode:

(a) exemple of time distribution of the chamber operating at $9 \mathrm{KV}$.

(b) Time resolution as a function of high voltage.

there is a tail of late signals which becomes more important for lower operating voltage, as pointed out before and for high fluxes. However with the time resolution measured for fluxes up to 250 $\mathrm{Hz} / \mathrm{cm}^{2}$ the $98 \%$ of events are included in a gate of $20 \mathrm{~ns}$, as it will be in the Alice experiment.

The big charge, generated inside the chamber by streamers ( $\sim 0.5 \mathrm{nC} /$ streamer $)$, affects the $\mathrm{RPC}$ performances and could produce a possible ageing effect of the chamber.

The high current which flow inside a large RPC 


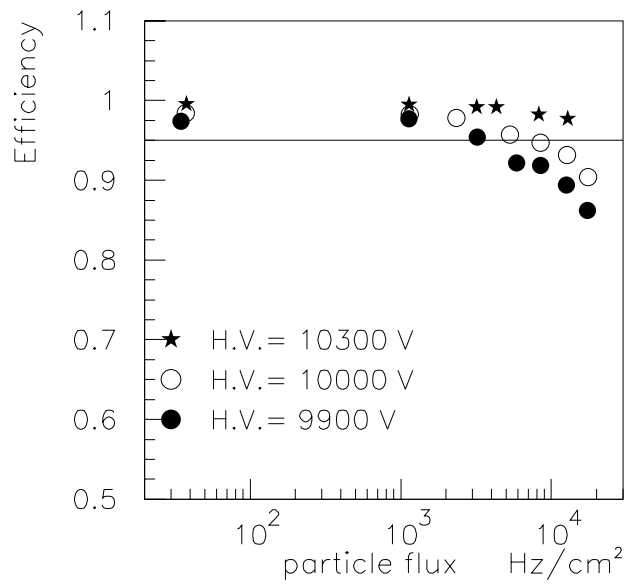

Figure 6. Efficiency of low resistivity RPC operating in avalanche mode as a function of particle flux for 3 different high voltage.

due to background particles in the Alice environement, could raise the electrode resistivity and then deteriorate the RPC performances after a long operation period. New tests are planned in order to study this effect.

\subsection{Avalanche mode results}

The avalanche mode is characterized by a saturated avalanche regime of the detector where the gas gain is not much dependent from the operating voltages, this region is not far from the streamer threshold and largely dependent from the gas mixture. The presence of streamers in RPC operating in avalanche mode must be reduced to very low value otherwise the large charge produced inside the chamber would increase the pick-up strip multiplicity deteriorating the cluster size. With the gas mixture used during the test, and with a discrimination threshold of 200 $\mathrm{mV}$, the efficiency plateau ( $\epsilon \geq 95 \%)$ starts at $9.8 \mathrm{KV}$ and the fraction of events with streamers was always very low, less than $4 \%$ for a $500 \mathrm{~V}$ wide voltage range. At an operation voltage of $10 \mathrm{KV}$ only $\sim 1 \%$ of events produced a streamer. The important advantage of the avalanche operation mode, with respect to the streamer operation mode, is the greater rate capability which
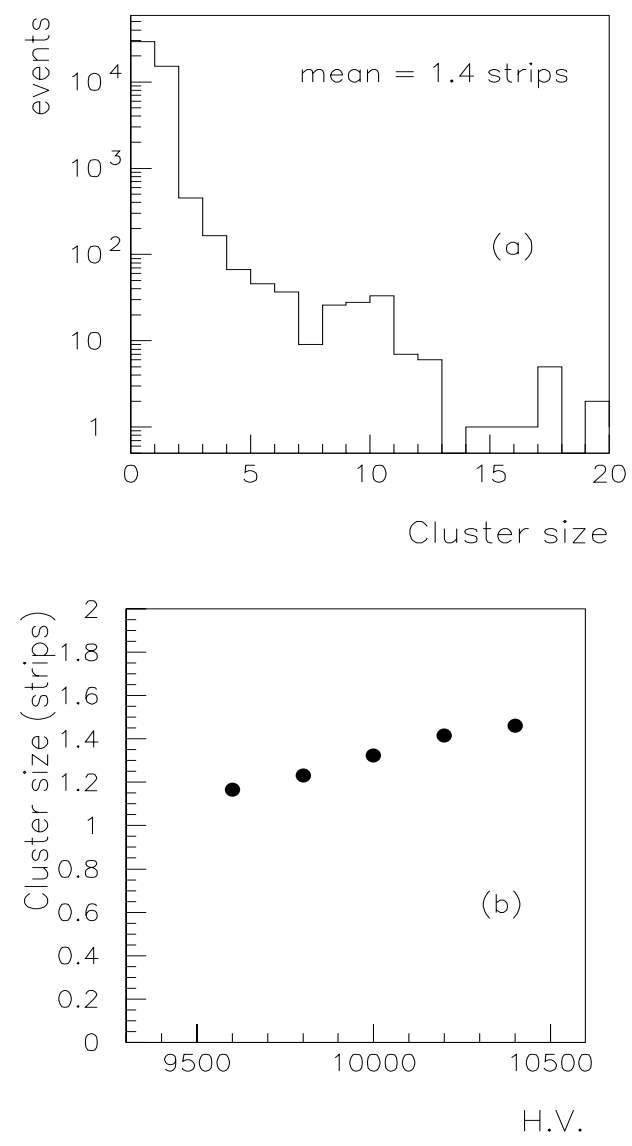

Figure 7. Cluster size mean value for the low resistivity RPC operating in avalanche mode:

(a) example of cluster size distribution for an operating voltage of $10 \mathrm{KV}$.

(b) mean cluster size as a function of operating voltage.

can reach values of several $\mathrm{KHz} / \mathrm{cm}^{2}$ as the charge produced is smaller. In fig. 6 the low resistivity chamber efficiency is plotted as a function of the particle flux for three operation voltage. The maximum flux tolerated by the chamber with $\epsilon \geq$ $95 \%$ is $3 \mathrm{KHz} / \mathrm{cm}^{2}, 7 \mathrm{KHz} / \mathrm{cm}^{2}$ and more than $10 \mathrm{KHz} / \mathrm{cm}^{2}$ respectively for $9.9 \mathrm{KV}, 10 \mathrm{KV}$ and $10.3 \mathrm{KV}$.

Even if the presence of streamers is very low, as mentioned before, the cluster size mean value is rather large: from 1.2 to 1.5 strips ( strip width 


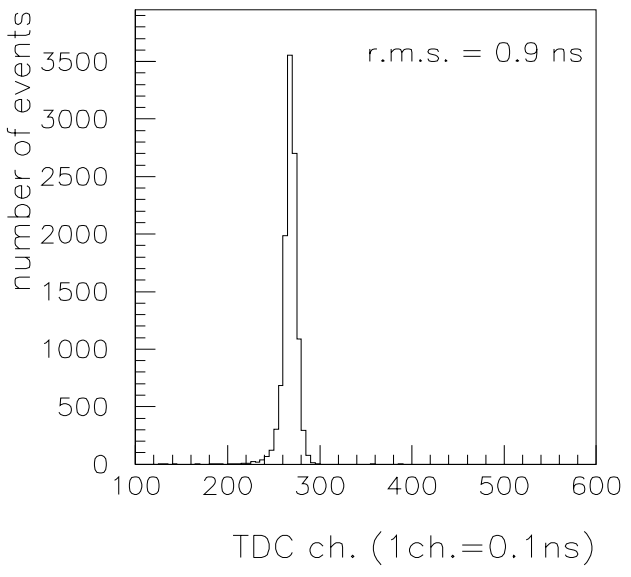

Figure 8. Example of time distribution for the low resistivity RPC operating in avalanche mode.

$=2 \mathrm{~cm}$ ), increasing with the operation voltage as shown in fig. $7 \mathrm{~b}$. An exemple of cluster size distribution is presented in fig. 7a for an operation voltage of $10 \mathrm{KV}$ and a particle flux of $1.1 \mathrm{KHz} / \mathrm{cm}^{2}$ : a mean value of 1.4 strips was obtained, this value has to be compared to 1.1 strips obtained in streamer mode. The larger cluster size is mainly due to electronic problem such as noise and cross-talk between strips.

The avalanche signals have a very small time fluctuation as shown in fig. 8 where an example of time distribution for the low resistivity chamber is presented: a r.m.s. of less than 1 ns was obtained. This value is quite stable and it is not dependent from the high voltage and the particle flux.

\section{CONCLUSION}

A study of RPC with surface of $50 \times 50 \mathrm{~cm}^{2}$ and with bakelite electrodes of different resistivity was performed at the SPS in order to define the parameters of the chambers which will be used in the Alice dimuon trigger. Rate capability, cluster size and time resolution were measured for the RPCs operating in streamer mode and in avalanche mode.

The low resistivity chamber $\left(\rho=3.5 \cdot 10^{9} \Omega \mathrm{cm}\right)$ tolerates fluxes up to $550 \mathrm{~Hz} / \mathrm{cm}^{2}$ with an efficiency $\geq 95 \%$ when it is operating in streamer mode, and fluxes up to $10 \mathrm{KHz} / \mathrm{cm}^{2}$ when it is operating in avalanche mode.

The mean cluster size (1.1 strips with $2 \mathrm{~cm}$ wide strips) and the time resolution (2.3 ns r.m.s.) of this RPC operating in streamer mode satisfy the Alice experimental requirements. Nevertheless ageing effects due to the high current flowing inside the chamber operating in streamer mode could deteriorate the RPC performances, therefore more tests are planned to study these effects. The performances of RPC operating in avalanche mode satisfy well the experimental requirements for what it concerns rate capability and time resolution, but a larger mean cluster size (1.4 strips) than that obtained in streamer operation mode was measured, this is mainly due to noise and cross-talk between the strips.

\section{REFERENCES}

1. T. Matsui and M. Satz, Phys. Lett. B178 (1986) 416.

2. Alice Collaboration, Addendum to the Technical Proposal CERN/LHCC 96-32 (1996).

3. R. Santonico et al. NIM 187 (1981) 377.

4. R. Cardarelli et al., NIM A263(1988).

5. A. Morsh, Alice note 96-29/dim(1996).

6. A. Baldit et al., Alice note $98-16 / \operatorname{dim}(1998)$.

7. P. Camarri et al.,Proceeding of IV International Workshop on RPC, Castel dell'Ovo, Napoli oct 15-16-1997. 